\title{
Iris Recognition: Measuring Feature's Quality for the Feature Selection in Unconstrained Image Capture Environments
}

\author{
Hugo Proença and Luís A. Alexandre \\ Dep. Informatics, IT - Networks and Multimedia Group \\ Universidade da Beira Interior, Covilhã, Portugal \\ Email: \{hugomcp,lfbaa\}@di.ubi.pt
}

\begin{abstract}
Iris recognition has been used for several purposes.However, current iris recognition systems are unable to deal with noisy data and substantially increase their error rates, specially the false rejections, in these conditions. Several proposals have been made to access image quality and to identify noisy regions in iris images. In this paper we propose a method that measures the quality of each feature of the biometric signature and takes account into this information to constraint the comparable features and obtain the similarity between iris signatures. Experiments led us to conclude that this method significantly decreases the error rates in the recognition of noisy iris images, resultant from capturing in less constrained environments.
\end{abstract}

Keywords- Feature Quality, Feature Comparison, Noncooperative Iris Recognition, Biometrics.

\section{INTRODUCTION}

Since 1987, when L. Flom and A. Safir concluded about the stability of iris morphology and estimated the probability for the existence of two similar irises at 1 in $10^{72}$, the use of iris based biometric systems has been increasingly encouraged by both government and private entities. Iris is commonly recognized as one of the most reliable biometric measures: it has a random morphogenesis and, apparently, no genetic penetrance. However, the error rates substantially increase, specially the false rejections, when the images do not have enough quality, either due to focus, contrast or brightness problems, iris obstructions or reflections, as exemplified by figure 1 .

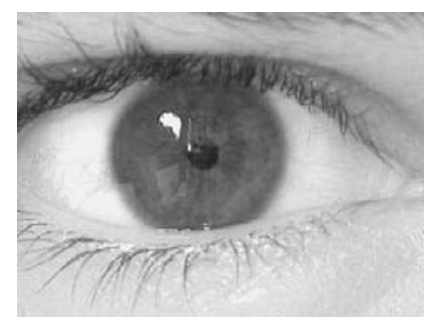

Fig. 1. Noisy iris image with eyelid and eyelash obstructions and large reflection areas
In this paper we propose a method for measuring the quality of each feature extracted from the iris. This measure is based on the proportion of noisy pixels used in the extraction of each feature and is further used in the feature comparison stage, to achieve robustness to noisy images. We used images from the UBIRIS [9] database to compare the results obtained by the classical Daugman recognition method [3] with and without our quality measure and comparison proposals.

The remainder of this paper is organized as follows: section II briefly summarizes the most cited iris recognition methods, emphasizing the feature extraction and comparison stages. A detailed description of the proposed method is given in section III. Section IV reports the experiments and results and, finally, section $\mathrm{V}$ concludes this paper.

\section{IRIS RECOGNITION}

In spite of the distinct approaches proposed by different authors for each stage, typical iris recognition systems share a common structure that is illustrated on figure 2 .

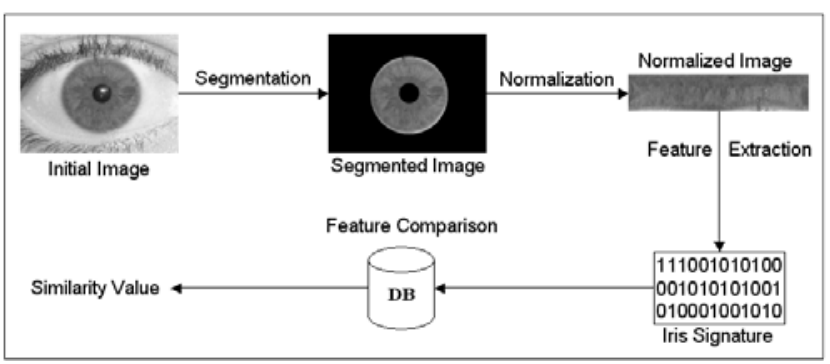

Fig. 2. Typical stages of the iris recognition

The initial stage deals with iris segmentation. This process consists in localize the iris inner (pupillary) and outer (scleric) borders. There are two major strategies for iris segmentation: using a rigid or deformable template of the iris or its boundary. In most cases, the boundary 
approach is very similar to the proposed by Wildes [12]: it begins by the construction of an edge map followed by the application of some geometric form fitting algorithm. The template-based strategies usually involve the maximization of some equation, as proposed by Daugman [3].

In order to compensate the varying size of the pupil it is common to translate the segmented iris part represented in the cartesian coordinate system to a fixed length and dimensionless polar coordinate system. This is usually accomplished through a method similar to the Daugman's Rubber Sheet [3].

From the feature extraction viewpoint, iris recognition approaches can be divided into three major categories: phasebased methods (e.g. [3]), zero crossing methods (e.g. [1]) and texture analysis based methods (e.g [12]).

After obtaining a feature set, commonly named biometric iris signature, the final stage consists in the comparison between signatures, producing a numeric dissimilarity value. If this value is higher than a threshold, the system outputs a "non-match", meaning that each signature belongs to different subjects. Otherwise, the system outputs a "match", meaning that both signatures were extracted from the same person.

\section{A. Noise Identification}

The problem of noise identification in iris images has been recently addressed by several authors, constituting one of the most challenging domains in the present iris recognition research.

Daugman [3] builds a binary noise mask for iris image to identify eyelids and eyelashes that obstruct iris and can deteriorate results.

In [7] there are extended previous research efforts on iris image quality assessment and analyzed the effect of seven quality factors: defocus blur, motion blur, off-angle, occlusion, specular reflection, lighting and pixel counts. Further, the authors describe methods for the estimation of global defocus, motion and offangle problems in the iris images.

The authors of [2] propose local and global iris image quality measures based in the analysis of the energy resultant from the convolution between the image and a group of Mexican-Hat Wavelets at 3 different scales.

The purpose of [5] and [10] was the identification of four distinct types of noise: eyelashes, eyelids, reflections and pupil. The idea is that there's always some type of edge between the noisy and the noise-free areas. Those edges were identified through an illumination invariant measure (phase congruency).

The later described methods operate locally and produce a binary map where each pixel is classified as "noisy" or "noise-free". Our feature quality measure is based in this binary map.

\section{PROPOSED FEATURE QUALITY MEASURE AND COMPARISON METHODS}

The local noise identification methods described in section II-A produce a binary map correspondent to the segmented and normalized iris image where the noisy regions appear as dark areas and the noise-free regions are represented through white areas (figure 3).

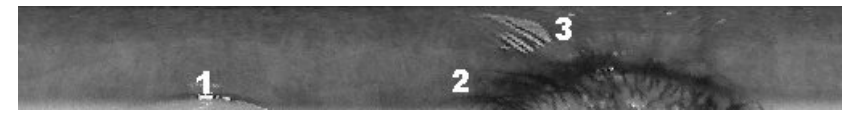

(a) Segmented and normalized iris image with large noisy regions correspondent to eyelids (1) and eyelashes (2) obstructions and reflections (3).

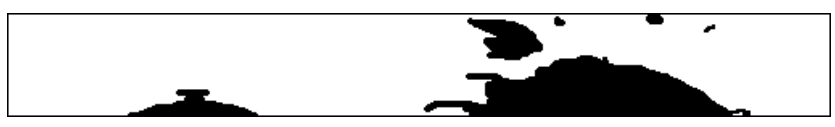

(b) Identification of the noisy regions from the image 3a.

Fig. 3. Identification of the noisy regions in the normalized iris image

For each of the extracted features from the image we create a quality value contained in the $[0,1]$ interval that reflects the proportion of noisy pixels used in the respective calculus. This value will be helpful in the feature comparison stage, avoiding the utilization of the noisiest features or assigning them a small weight for the comparison.

As described in the next section, the proposed feature quality measure is independent from the feature extraction method. At a coarse level, every extracted feature can be regarded as function of $\mathrm{K}$ pixels from the image (the original data). Thus, the calculus of the proportion of noisy and noise-free pixels used in its extraction can be applied to any feature extraction methodology.

\section{A. Feature Quality Measure}

Let $I$ be the segmented and normalized iris image and $(x, y) \in N^{2}$ be the coordinates of a pixel from the image. Let $n((x, y)): N^{2} \rightarrow\{0,1\}$ be the function that classifies as "noisy" or "noise-free" every pixel $(x, y)$ from the image $I$ :

$n(x, y)=\left\{\begin{array}{l}1, I(x, y) \text { noisy } \\ 0, \text { otherwise }\end{array}\right.$

Let $F=\left\{f_{1}, \ldots, f_{k}\right\}$ be the set of features extracted from the image $I$. At the coarsest level, every $f_{i}$ is obtained using $N_{i}$ pixels from $I$, independently of the used feature extraction method. Let $P i=\left\{\left(x_{i j}, y_{i j}\right)\right\} j=1, \ldots, N_{i}$ be the set of pixels' coordinates used in the extraction of the feature $\mathrm{f}_{\mathrm{i}}$. We define the function $q\left(P_{i}\right):\left(N^{2}{ }_{1} \times \ldots \times N^{2}{ }_{N}\right)$ $\rightarrow[0,1]$ that gives the quality of the feature fi: 


$$
q\left(P_{i}\right)=\frac{1}{N} \sum_{i=1}^{N_{i}} n\left(I\left(x_{i}, y_{i}\right)\right)
$$

This function $\mathrm{q}($.$) gives the proportion of noisy data$ considered in the extraction of fi. Through this, every extracted feature has a quality value contained in the $[0,1]$ interval. This value has inverse correspondence with the proportion of noisy pixels evolved in the creation of fi. Thus, for completely "noisy" (poorest quality) and "noisefree" (optimal quality) features, the quality value will be respectively equal to 0 and 1 .

\section{B. Feature Comparison}

In the following discussion we will use a superscript to distinguish between two different feature sets, such as, $F^{1}$ and $F^{2}$, and a subscript to distinguish between different features from a feature set, such as, $f_{1}^{l}$ and $f_{2}^{1}$.

The purpose of the feature comparison stage is to obtain a similarity value between two feature sets $-F_{i}$ and $F_{j}$ - that enables the assumption about the identity of the subjects from which they were extracted. In this section, we describe the two tested variants for the feature comparison stage: "hard" and "fuzzy".

In the "hard" feature comparison, features are considered for comparison if their quality value is higher than a threshold. Oppositely, in the "fuzzy" feature comparison the comparison is allowed for all features independently of their quality value, although the result is weighted with the average quality value from the feature operands.

Formally, let $Q^{0}$ and $Q^{1}$ be two features sets with the respective quality values for each feature: $Q^{j}=\left\{\left(f_{1}{ }^{j}, q\left(f_{1}\right.\right.\right.$ $\left.\left.\left.{ }^{j}\right)\right), \ldots,\left(f_{n}{ }^{j}, q\left(f_{n}{ }^{j}\right)\right)\right\}$. Our proposed feature comparison function is given by:

$$
f_{C}\left(Q^{0}, Q^{1}\right)=\sum_{i=1}^{n} \frac{\operatorname{dist}\left(f_{i}^{0}, f_{i}^{1}\right)}{\operatorname{cnt}\left(f_{i}^{0}, f_{i}^{1}\right)}
$$

In the "hard" comparison variant, dist and cnt are the functions that respectively give the similarity between two features and information about feature comparability. They are given by:

$$
\begin{aligned}
& \text { dist }_{\text {hard }}\left(f_{i}^{0}, f_{i}^{1}\right)=\left\{\begin{array}{l}
d\left(f_{i}^{0}, f_{i}^{1}\right), q\left(f_{i}^{0}\right) \wedge q\left(f_{i}^{1}\right) \geq T \\
0, \text { otherwise }
\end{array}\right. \\
& \text { cnt }_{\text {hard }}\left(f_{i}^{0}, f_{i}^{1}\right)=\left\{\begin{array}{l}
1, q\left(f_{i}^{0}\right), q\left(f_{i}^{1}\right) \geq T \\
0, \text { otherwise }
\end{array}\right.
\end{aligned}
$$

where $d($.$) is the function that gives the distance between$ features (e.g. Hamming, Euclidean distance) and $\mathrm{T}$ is a threshold value in $[0,1]$.

In the "fuzzy" comparison variant, all the features are considered for comparison and weighted according to their quality value. Thus, dist and cnt are given by:

$$
\begin{aligned}
& \operatorname{dist}_{\text {fuzzy }}\left(f_{i}^{0}, f_{i}^{1}\right)=d\left(f_{i}^{0}, f_{i}^{1}\right) * \frac{q\left(f_{i}^{0}\right)+q\left(f_{i}^{1}\right)}{2} \\
& \operatorname{cnt}_{\text {fuzzy }}\left(f_{i}^{0}, f_{i}^{1}\right)=\frac{q\left(f_{i}^{0}\right)+q\left(f_{i}^{1}\right)}{2}
\end{aligned}
$$

where, as above, $d($.$) is the function that gives the distance$ between features.

\section{EXPERIMENTS AND DISCUSSION}

We implemented the method described by Daugman [3]. We compared the obtained results when following the method as described by the author (using all the features in the feature comparison stage) and our proposal feature quality measure and feature comparison methods.

As described in [3], the Daugman's recognition method is composed by the following stages:

- Iris segmentation. We implemented the integrodifferential operator proposed by the author to find both the inner and outer iris borders, given by:

$$
\max _{r, x_{0}, y_{0}}\left|G_{\sigma}(r) * \frac{\delta}{\delta_{r}} \oint_{r, x_{0}, y_{0}} \frac{I(x, y)}{2 \Pi r} d s\right|
$$

This operator searches over the image domain $(x$, $y$ ) for the maximum in the blurred partial derivative with respect to increasing radius $r$, of the normalized contour integral of $I(x, y)$ along a circular arc $d s$ of radius $\mathrm{r}$ and center coordinates $\left(x_{0}, y_{0}\right)$.

- Normalization. To compensate the variations in the size of the pupil, we translated the images to dimensionless polar coordinate system through a process known as the "Daugman Rubber Sheet" [3].

- Feature Extraction. The iris data encoding was accomplished through the use of two dimensional Gabor filters. These spatial filters have the form:

$$
\begin{aligned}
& G(x, y)=e^{-\pi\left[\left(x-x_{0}\right)^{2} / \alpha^{2}+\left(y-y_{0}\right)^{2} / \beta^{2}\right]} \\
& * e^{-2 \pi i\left[\left(x-x_{0}\right) u_{0}+\left(y-y_{0}\right) v_{0}\right]}
\end{aligned}
$$


where $\left(\mathrm{x}_{0}, \mathrm{y}_{0}\right)$ defines the position in the image, $(\alpha, \beta)$ is the filter width and length and $\left(u_{0}, v_{0}\right)$ specify the modulation, having spatial frequency $\mathrm{w}_{0}=\operatorname{sqrt}\left(\mathrm{u}_{0}^{2}+\mathrm{v}_{0}^{2}\right)$ and direction $\theta_{0}=\arctan \left(\mathrm{v}_{0} / \mathrm{u}_{0}\right)$.

The real parts of the 2-D Gabor filters are truncated to be zero volume and achieve illumination invariance. For each resulting bit we analyzed the sign of the real and imaginary parts from quadrature image projections and through quantization assigned binary values: 1 and 0 respectively for positive and negative projection values.

- Feature Comparison. The feature extraction binarization process allows the utilization of the Hamming distance as the similarity measure for two iris signatures. Given two binary sets with $N$ bits: $A=\left\{a_{1}, \ldots, a_{N}\right\}$ and $B=\left\{b_{1}, \ldots, b_{N}\right\}$, the Hamming distance is given by the sum of the logical XOR operation between each pair $\left(a_{i}, b_{i}\right)$.

The identification of the noisy pixels in the normalized iris image was accomplished following the method described in [10], that produces a binary map with the noisy pixels correspondent to the dark regions, as illustrated by figure $3 b$.

Through the process described in section III we obtain a quality measure for each feature of the biometric signature. Hereinafter, the task consists in determining the features that must be compared in order to maximize the separability between the intra- and inter-class comparisons (respectively resultant from images from the same and different irises).

In the limit, if all the features are compared, the probability that some of these features are corrupted by noise and deteriorate the results is maximized. Oppositely, if the feature comparison is exclusively made between features with optimal quality value (completely noisefree), the number of comparisons is minimized. Given the potentially small number of completely noise-free features, the random similarities or dissimilarities between iris signatures (that is always present) may also deteriorate the final results. Thus, the number of comparable features must not be very large (we want to avoid

features that are corrupted by noise), but must also not be very small (to avoid the sensibility to the random nature of the features).

As we describe in the next sections, we will empirically choose the value for the minimum quality of the comparable features to match these criterions. In the experiments, we varied both the feature comparison variant and the minimum quality value demanded to compare features and analyzed the recognition accuracy in two distinct data sets with different noise quantities.

\section{A. Data Sets}

In this section we present the available data sets and justify our choice for the data sets used in our experiments.

There are presently 5 public and freely available iris image databases for biometric purposes: CASIA [6], MMU [8], BATH [11], UPOL [4] and UBIRIS [9].

CASIA database is by far the most widely used for iris biometric purposes. However, its images incorporate few types of noise, almost exclusively related with eyelid and eyelash obstruction, as the images from MMU and BATH databases. UPOL images were captured with an optometric framework, obtaining optimal images with extremely similar characteristics. Oppositely, UBIRIS database was builded with the objective of simulate non-cooperative image capturing. This fact explains the higher heterogeneity of its images and make it the most appropriate for the objectives of our work.

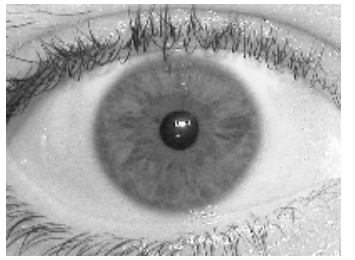

(a) Good quality iris image

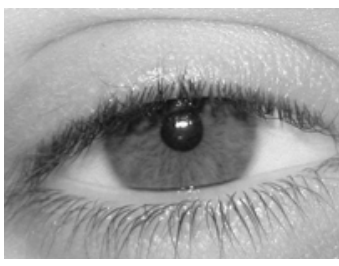

(c) Iris with eyelids obstructions

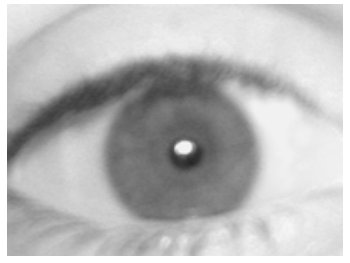

(b)Poor foccused iris image

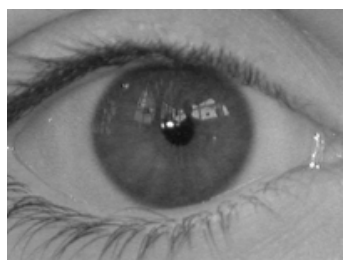

(d) Eye with reflections
Fig. 4. Examples of images from the UBIRIS database

We selected 260 images from the UBIRIS database, belonging to 26 different subjects (10 images from each subject). Figure 4 contains examples from the images used in our experiments. However, we considered relevant the information about the recognition's accuracy as the amount of noise in the image varies. In order to enable this analysis, we further divided the selected images in two sub sets, according to their noise characteristics. The 130 less noisy images were included in the UBIRIS $_{\mathrm{F}}$ data set and the 130 noisier ones in the UBIRIS $_{\mathrm{N}}$ data set.

\section{B. Results}

According to the process described in the beginning of section IV, we compared the accuracy of the classical Daugman methodology as described by the author and together with our proposals, in the above described data sets $\left(\right.$ UBIRIS $_{\mathrm{F}}$ and UBIRIS $_{\mathrm{N}}$ ). We made the feature extraction for every image and compared the resultant 
feature set with all the remaining feature sets of the same data set.

Figure 5 contains 4 measures that reflect the advantages of applying our proposal together with the classical Daugman feature extraction and comparison methods. The horizontal axis represents the minimum feature quality value using the "hard" comparison variant. The continuous line is relative to the UBIRIS $_{\mathrm{N}}$ data set and the dashed line to the less noisier UBIRIS $_{\mathrm{F}}$ data set.

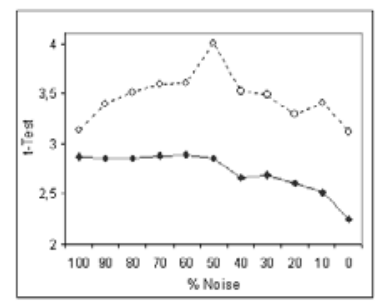

(a) t-Test values

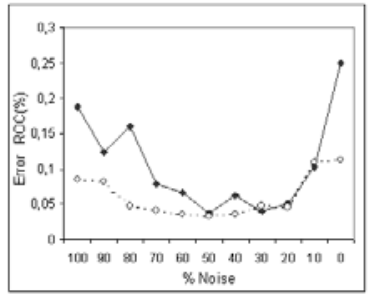

(c) Area under the ROC

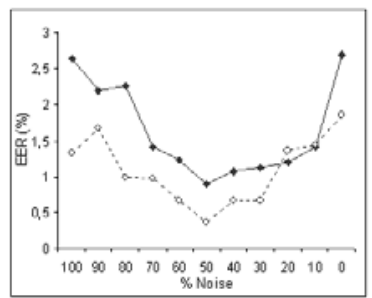

(b) Equal error rate

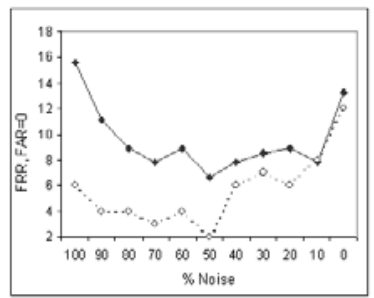

(d) FRR, FAR=0
Fig. 5. Obtained results from our proposal in the UBIRISN (continuous line) and UBIRISF (dashed line) by:

Figure 5a contains the obtained values for a t-test given

$$
\tau=\frac{\mu^{E}-\mu^{I}}{\sqrt{\frac{\sigma^{I 2}}{N^{I 2}}+\frac{\sigma^{E 2}}{N^{E 2}}}}
$$

where symbols $\mu^{\mathrm{I}}$ and $\mu^{\mathrm{E}}$ respectively indicate the obtained means in the intra-class (images from the same iris) and interclass (images from different irises) comparisons. $\sigma^{\mathrm{I}}$ and $\sigma^{\mathrm{E}}$ indicated the respective standard deviations and $\mathrm{N}^{\mathrm{I}}$ and $\mathrm{N}^{\mathrm{E}}$ are, respectively, the total number of intra-class and inter-class comparisons between iris signature.

Figure $5 \mathrm{~b}$ contains the equal error rates, obtained when the false accepts and rejects are approximately equal. Figure 5c contains the percent values for the area under the receiver operating curve (ROC) and figure $5 \mathrm{~d}$ contains the obtained value for the false rejections, when the false acceptances are minimized.

The best results were obtained when the feature comparison was enabled between features with minimum quality value around $0.5(50 \%)$. In this situation, while maintaining a large number of comparable features, very poor quality features, extracted from large proportion of noisy pixels, are not compared and do not corrupt the final results. Our experiments clearly show an improvement of the system's accuracy, significantly reducing the error rates, the area under the ROC and increasing the separability between the intra-class inter-class comparisons.

\begin{tabular}{|c|c|c|c|c|}
\hline Method & $\tau$ & EER (\%) & $\begin{array}{c}\text { Error ROC } \\
(\%)\end{array}$ & $\begin{array}{c}\text { FRR, FAR=0 } \\
(\%)\end{array}$ \\
\hline \begin{tabular}{c} 
UBIRIS data set \\
\hline Original
\end{tabular} & 2.258 & $2.090 \pm 0.004$ & 0.449 & $15.556 \pm 0.010$ \\
\hline $\begin{array}{c}\text { Proposed (hard), } \\
\text { T=0.5 }\end{array}$ & 2.892 & $0.904 \pm 0.002$ & 0.036 & $6.667 \pm 0.007$ \\
\hline Proposed (fuzzy) & 2.699 & $1.130 \pm 0.002$ & 0.057 & $11.639 \pm 0.009$ \\
\hline UBIRIS data set \\
\hline Original & 3.130 & $2.690 \pm 0.004$ & 0.112 & $12.778 \pm 0.009$ \\
\hline $\begin{array}{c}\text { Proposed (hard), } \\
\text { T=0.5 }\end{array}$ & 4.012 & $0.366 \pm 0.001$ & 0.032 & $2.667 \pm 0.004$ \\
\hline Proposed (fuzzy) & 3.813 & $0.900 \pm 0.002$ & 0.058 & $6.166 \pm 0.006$ \\
\hline
\end{tabular}

Table 1. Results obtained by the Daugman recognition method with and without our method proposal.

Table I contains the obtained results, when varying the feature comparison strategy. The first column identifies the classification method. The second $(\tau)$ contains the value for the t-test described above, the third column the equal error rate and the last column the obtained false rejection rate when the false acceptances were minimized. All the error values are expressed for a confidence interval of $95 \%$.

The analysis of these results clearly shows an increment of system's overall accuracy using our proposal - with the "hard" variant, specially in the noisier UBIRIS $_{N}$ data set. In the less-noisier UBIRIS $_{\mathrm{F}}$ data set the results were more similar, meaning that even in images with insignificant portions of noise our proposal can slightly improve the recognition's accuracy.

With the "fuzzy" feature comparison variant, we obtained worst results than with the "hard" one. The comparison of all the features, even if they are weighted with their quality value, is not a valid alternative.

\section{CONCLUSIONS}

In this paper we proposed a method for the measurement of the quality of each extracted feature for iris recognition. The motivation for this work was the significant increment of the error rates when these recognition systems deal with noisy images.

The quality value is used to constraint the number of features that are taken into account in the feature comparison stage. Experiments led us to conclude that this method significantly decreases the error rates in the recognition of noisy iris images, thus being appropriate for the application in a non-cooperative recognition compass, where the ability to deal with noisy images is required. 
Even in images with better quality, with minor portions of noise, the results showed that our quality measure contributes for the selection of the best features with the correspondent improvement in the system's recognition accuracy. Moreover, the independency of our proposal from the choused feature extraction and comparison methodology can be regarded as a strong point.

\section{REFERENCES}

[1] W. W. Boles and B. Boashash. A human identification technique using images of the iris and wavelet transform. IEEE Transactions on Signal Processing, vol. 46, no. 4, pages 1185-1188, April 1998.

[2] Yi Chen, Sarat C. Dass, and Anil K. Jain. Localized iris image quality using 2-D wavelets. In Proceedings of the 2006 International Conference on Biometric, pages 373-381, Hong Kong, 2006.

[3] John G. Daugman. High confidence visual recognition of persons by a test of statistical independence. IEEE Transactions on Pattern Analysis and Machine Intelligence, Vol. 25, No. 11, pages 1148-1161, November 1993.

[4] Michal Dobes and Libor Machala. UPOL iris image database, 2004. http://phoenix.inf.upol.cz/iris/

[5] Junzhou Huang, Yunhong Wang, Tieniu Tan, and Jiali Cui. A new iris segmentation method for recognition. In Proceedings of the 17th
International Conference on Pattern Recognition (ICPR04), vol. 3, pages 23-26, 2004.

[6] Institute of Automation, Chinese Academy of Sciences. CASIA iris image database, 2004. http://www.sinobiometrics.com

[7] Nathan Kalka, Jinyu Zuo, Natalia Schmid, and Bojan Cukic. Image quality accessment for iris biometric. In Proceedings of the SPIE Biometric Technology for Human Identification III, vol. 6202, pages 263-273, April 2006.

[8] Multimedia University. MMU iris image database, 2004. http://pesona.mmu.edu.my/ccteo

[9] Hugo Proença and Luís A. Alexandre. UBIRIS: A noisy iris image database. In 13th International Conference on Image Analysis and Processing (ICIAP2005), pages 970-977, September 2005. http://iris.di.ubi.pt

[10] Hugo Proença and Luís A. Alexandre. A method for the identification of noisy regions in normalized iris images. In IEEE Proceedings from the 18th International Conference on Pattern Recognition - (ICPR2006), vol 4, pages 405-408, , August 2006.

[11] University of Bath. University of Bath iris image database, 2004. www.bath.ac.uk/elec-eng/pages/sipg/

[12] Richard P. Wildes. Iris recognition: an emerging biometric technology. In Proceedings of the IEEE, vol. 85, no.9, pages 1348-1363, U.S.A., September 1997. 